\title{
Glutamatergic Inputs Contribute to Phasic Activity in Vasopressin Neurons
}

\author{
Jean-Marc Israel, ${ }^{1,2}$ Dominique A. Poulain, ${ }^{1,2}$ and Stéphane H. R. Oliet ${ }^{1,2}$ \\ ${ }^{1}$ Inserm Unité 862, Neurocentre Magendie, and 2Université de Bordeaux, 33077 Bordeaux, France
}

\begin{abstract}
Many neurons in the CNS display rhythmic patterns of activity to optimize excitation-secretion coupling. However, the mechanisms of rhythmogenesis are only partially understood. Magnocellular vasopressin (VP) neurons in the hypothalamus display a phasic activity that consists of alternative bursts of action potentials and silent periods. Previous observations from acute slices of adult hypothalamus suggested that VP cell rhythmicity depends on intrinsic membrane properties. However, such activity in vivo is nonregenerative. Here, we studied the mechanisms of VP neuron rhythmicity in organotypic slice cultures that, unlike acute slices, preserve functional synaptic connections. Comparative analysis of phasic firing of VP neurons in vivo, in acute slices, and in the cultures revealed that, in the latter, the activity was closely related to that observed in vivo. It was synaptically driven, essentially from glutamatergic inputs, and did not rely on intrinsic membrane properties. The glutamatergic synaptic activity was sensitive to osmotic challenges and $\kappa$-opioid receptor activation, physiological stimuli known to affect phasic activity. Together, our data thus strongly suggest that phasic activity in magnocellular VP neurons is controlled by glutamatergic synaptic inputs rather than by intrinsic properties.
\end{abstract}

\section{Introduction}

Vasopressin (VP) plays a key role in body fluid and cardiovascular homeostasis. VP is synthesized in magnocellular neurons that accumulate in the supraoptic nucleus $(\mathrm{SON})$ and paraventricular nucleus of the hypothalamus. These neurons project to the neurohypophysis where VP is released into the blood. In normally hydrated, unstimulated rats, most VP neurons display a slow irregular pattern of electrical activity. Nevertheless, $\sim 10 \%$ display a characteristic phasic pattern comprised of bursts of firing interspersed by silent periods (Poulain and Wakerley, 1982; Hussy et al., 1997; Gouzènes et al., 1998). Hyperosmotic stimulation initiates (in nonspontaneously phasic neurons) or reinforces (in spontaneously phasic neurons) this phasic activity (Poulain et al., 1977; Wakerley et al., 1978), thus optimizing the efficiency of stimulus-secretion coupling (Dutton and Dyball, 1979). The interspike intervals in a burst of action potentials (APs) and the silent intervals between bursts are important determinants for neuropeptide release (Cazalis et al., 1985), and understanding the mechanisms underlying this specific pattern of activity is of crucial importance in the study of neurosecretion.

Most of our knowledge in this domain arises from experiments performed in acute hypothalamic slices or from explants, in which the phasic activity is seen to be entirely supported by nonsynaptic depolarizing afterpotentials (DAPs) after single

Received June 22, 2009; revised Nov. 12, 2009; accepted Nov. 21, 2009.

This work was supported by grants from Inserm, Université de Bordeaux, and Conseil Régional d'Aquitaine. We thank Drs. P. Ciofi, D. T. Theodosis, and D. L. Voisin for helpful discussion and critical reading of this manuscript, Prof. M. C. Lombard for her expertise in statistical analysis, and N. Dupuy for technical assistance.

Correspondence should be addressed to Jean-Marc Israel, Inserm Unité 862, Neurocentre Magendie, 146, rue Léo-Saignat, 33077 Bordeaux, France. E-mail: jean-marc.israel@inserm.fr.

DOI:10.1523/JNEUROSCI.2948-09.2010

Copyright $\odot 2010$ the authors $\quad 0270-6474 / 10 / 301221-12 \$ 15.00 / 0$ spikes (Andrew and Dudek, 1984; Armstrong et al., 1994; Ghamari-Langroudi and Bourque, 1998) whose summation mediates a plateau-like potential (Andrew and Dudek, 1983). Bursts appear to result, therefore, from a regenerative mechanism attributable to the proximity of spike threshold (Roper et al., 2004; Brown and Bourque, 2006). Accordingly, blockade of synaptic transmission in acute slices did not alter phasic firing (Hatton, 1982). However, in this preparation, synaptic activity is mostly impaired and usually reduced to miniature synaptic currents/ potentials (Wuarin and Dudek, 1993; Brussaard et al., 1996, 1997; Kabashima et al., 1997; Kombian et al., 2000), probably because axon terminals are severed from their glutamatergic cell bodies. In vivo, application of glutamate receptor antagonists like ketamine (Nissen et al., 1994), D (-)-2-amino-5-phosphonopentanoic acid (D-AP-5) (Nissen et al., 1995; Moos et al., 1997), 6-cyano-7nitroquinoxaline-2,3-dione (CNQX) (Nissen et al., 1995), or kynurenic acid (Brown et al., 2004) blocks phasic activity in VP neurons, suggesting that glutamatergic inputs play a key role in governing phasic activity. It is still not clear why this is not the case in vitro.

In the present study, we compared phasic activity in VP neurons in three different preparations: in lactating females in vivo, in acute hypothalamic slices from adult females, and in organotypic slice cultures in which glutamatergic inputs are preserved (Jourdain et al., 1999). We found that VP neuron phasic activity in organotypic cultures is more closely related to that displayed in vivo than the phasic pattern observed in acute slices. Furthermore, the rhythmic behavior displayed by VP neurons in organotypic cultures does not depend on DAPs but is driven by glutamatergic synaptic activity. Our observations thus question the importance of intrinsic properties as the basis of phasic activity in VP neurons and highlight the importance of extrinsic glutamatergic drive in promoting such activity. 

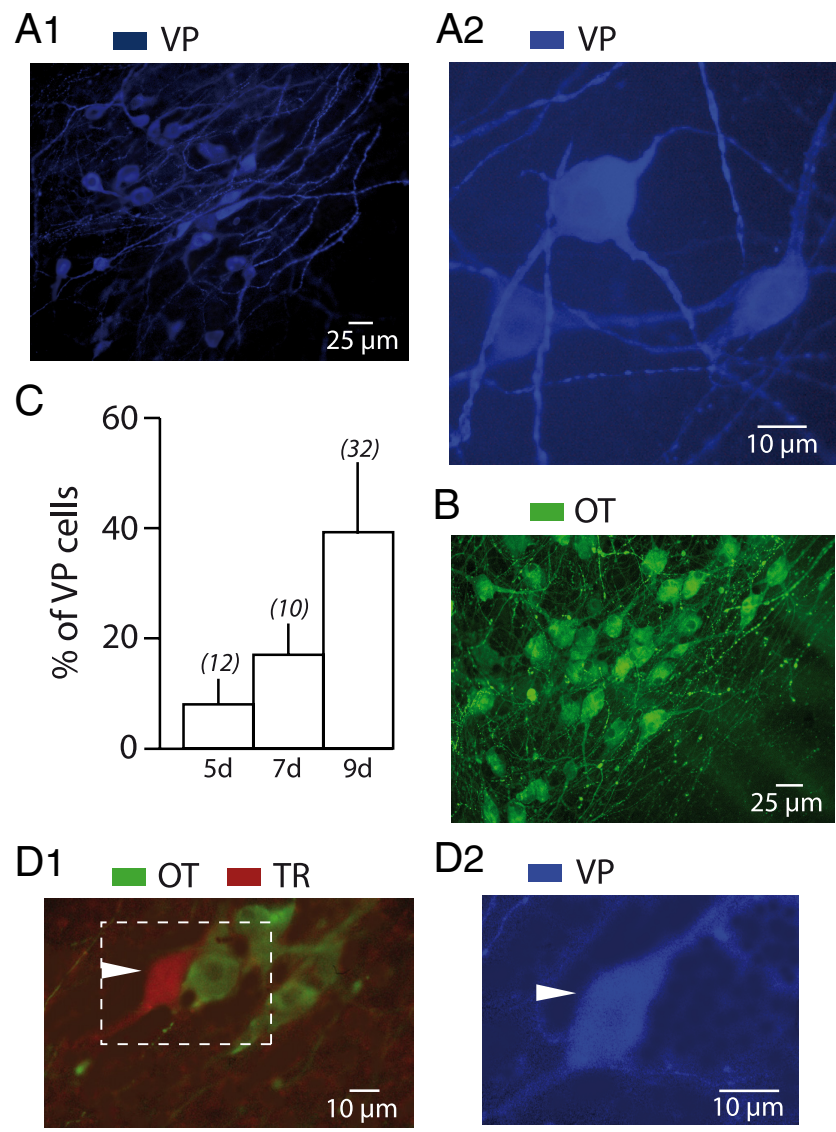

Figure 1. Vasopressin neurons in hypothalamic organotypic cultures. A, Example of an organotypic slice culture from the hypothalamus of a 9-d-old rat after 2 months in culture showing numerous VP (blue fluorescence) neurons at low (A1) and high magnification (A2). The magnocellular neurons generally possessed an ovoid or a round soma from which arose two to four thick dendritic-like processes. $\boldsymbol{B}$, Organotypic slice culture obtained from the hypothalamus of a 9-d-old rat after 2 months in culture. Immunocytochemical labeling revealed that these cultures are also enriched with $0 T$ (green fluorescence) neurons. C, Summary histogram depicting the percentage of VP-immunoreactive neurons in organotypic slice cultures obtained from 5- (5d), 7- (7d), and 9-d-old (9d) rat hypothalami, after a minimum of $40 \mathrm{~d}$ in culture. The number of experiments is indicated in brackets. $\boldsymbol{D}$, Example of a magnocellular neuron identified with biocytin (TR) (red fluorescence) after intracellular recording. Subsequent immunocytochemistry revealed that it displayed immunoreactivity for VP (blue fluorescence) (D2) but not for $0 \mathrm{~T}$ (green fluorescence) (D1).

\section{Materials and Methods}

Organotypic slice cultures. Organotypic slice cultures that included the SON were obtained from 5- to 9-d-old rat hypothalami. The cultures were prepared using the roller tube method, as described in detail by Jourdain et al. (1996). Briefly, Wistar rat pups were anesthetized with 5\% isoflurane $\left(95 \% \mathrm{O}_{2}\right)$ for $1 \mathrm{~min}$ and decapitated. Brains were removed and tissue blocks that included the hypothalamus were quickly dissected and sectioned $(400 \mu \mathrm{m})$ with a McIlwain tissue slicer. Frontal slices containing the SON were cut into two parts along the third ventricle, and each part was placed on a glass coverslip coated with heparinized chicken plasma. Thrombin was added to coagulate the plasma and permit adhesion of the slice to the coverslip. The coverslip was inserted into a plastic flat-bottom tube (Nalge Nunc International) containing $750 \mu \mathrm{l}$ of medium, pH 7.4 (290-295 mOsm), composed of 50\% Eagle's basal medium (Invitrogen), 25\% heat-inactivated horse serum (Invitrogen), and 25\% HBSS (Invitrogen) enriched with glucose $(7.5 \mathrm{mg} / \mathrm{ml})$; L-glutamate (Seromed) was added at a concentration of $2 \mathrm{~mm}$. No antibiotics were used. The tubes were tightly capped and inserted in a roller drum and rotated $\sim 15$ turns/h. The medium was replaced twice a week.

Using immunocytochemistry, we confirmed previous observations (Wray et al., 1991; Jourdain et al., 1996) showing that slice cultures
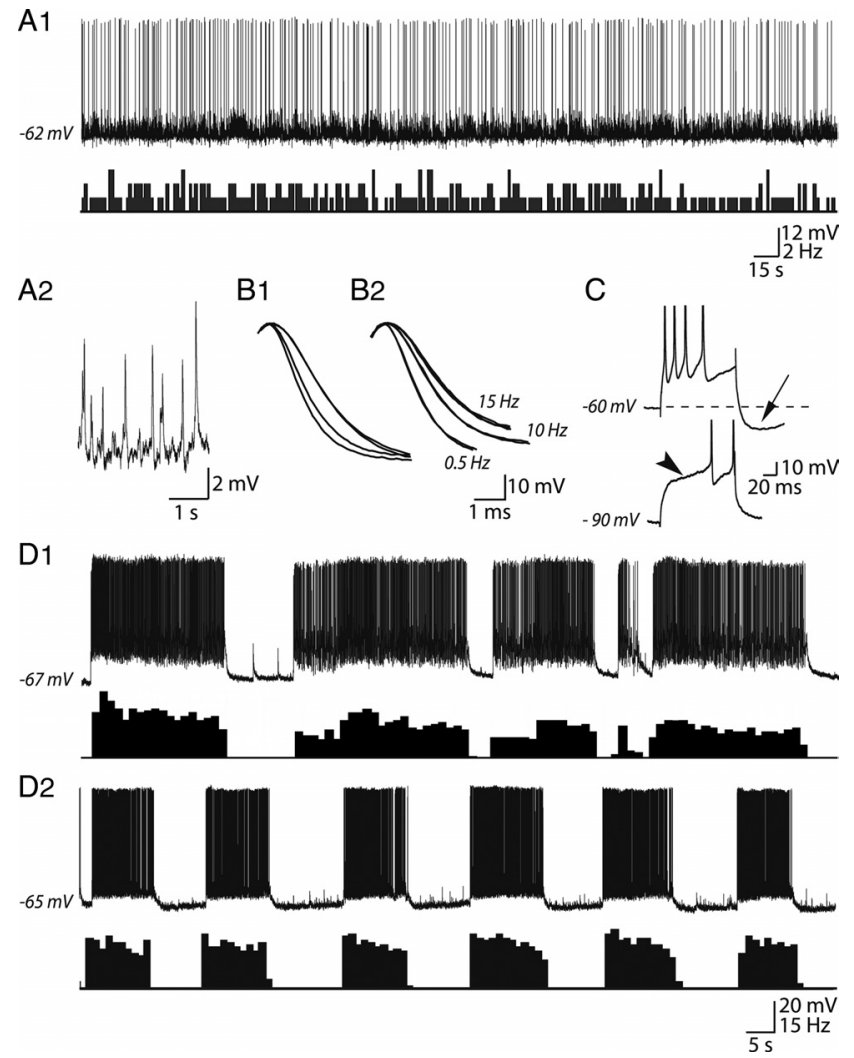

Figure 2. Basal electrical properties of VP neurons in organotypic slice cultures. A1, The top trace shows an intracellular recording from a VP neuron in a 10-week-old culture from a 9-d-old rat. The neuron displayed a slow irregular activity varying from 0.2 to $3 \mathrm{~Hz}$ as indicated by the sequential distribution histogram of AP discharge (bottom trace). In addition to APs, a sustained synaptic activity was clearly visible ( $\boldsymbol{A} 2)$. $\boldsymbol{B}$, Example showing the progressive duration increase (broadening) of APs during a burst (B1). The broadening was also frequency dependent as illustrated in $B 2$ in which APs were triggered by positive current pulses ( $+50 \mathrm{pA}, 50 \mathrm{~ms}$; in the presence of $(\mathrm{CNQX})$ at increasing frequencies $(0.5,10$, and $15 \mathrm{~Hz})$. C, Injection of a depolarizing current pulse (+70 pA, $100 \mathrm{~ms})$ (data not shown) applied at resting potential $(-60 \mathrm{mV})$ triggered a burst of APs. Note the presence of an afterhyperpolarizing potential (AHP) after the train of APs (arrow). When the cell was hyperpolarized to $-90 \mathrm{mV}$, a notch (arrowhead) delayed the occurrence of APs. $\boldsymbol{D}$, Two examples of spontaneous phasic activity characterized by successive bursts of APs and silent periods (D1, D2, top traces). The bottom traces are the corresponding sequential distribution histogram of APs, typical of phasic activity. Note the synaptic activity visible between bursts in $\mathbf{D 2}$.

derived from 5 -d-old pups are poor in VP neurons ( $8 \pm 4 \%$ of the total number of magnocellular cells; $n=12$ ) (Fig. 1C). However, their numbers increase in slice cultures from older pups: $17 \pm 6 \%$ in slices $(n=10)$ from 7 -d-old rats and $39 \pm 13 \%$ in slices $(n=32)$ from 9 -d-old pups (Fig. 1 $A 1, A 2, C$ ). We therefore performed our recordings in 4 - to 10 week-old slice cultures from hypothalamus of 9-d-old rats. Just before a recording session, a slice was transferred from the incubator to a temperature-controlled chamber $\left(36 \pm 0.2^{\circ} \mathrm{C}\right)$ fixed to the stage of an inverted microscope (Diaphot; Nikon). Magnocellular neurons were visualized by their large size. The microelectrode was positioned close to the cell to be recorded using oleic micromanipulators (Narishige).

For recording, the slices were perfused $(0.7 \mathrm{ml} / \mathrm{min})$ with Yamamoto's solution (in mm: $125 \mathrm{NaCl}, 3 \mathrm{KCl}, 1 \mathrm{MgSO}_{4}, 1.25 \mathrm{KH}_{2} \mathrm{PO}_{4}, 5 \mathrm{NaHCO}_{3}, 2$ $\mathrm{CaCl}_{2}, 5$ glucose, 10 HEPES, pH 7.25, 293-295 mOsm). Hyperosmolarity was produced by addition of mannitol, whereas hypo-osmotic medium was obtained by diminishing HEPES from 10 to $5 \mathrm{~mm}$. The following were added to the medium when required: CNQX (RBI), D-AP-5 (RBI), tetrodotoxin (TTX), flufenamic acid (FFA), dynorphine (SigmaAldrich), and nor-binaltorphimine (BNI) (Tocris Bioscience). Intracellular microelectrodes were pulled from borosilicated glass capillaries (outer diameter, $1 \mathrm{~mm}$; inner diameter, $0.5 \mathrm{~mm}$; Sutter Instrument) with 


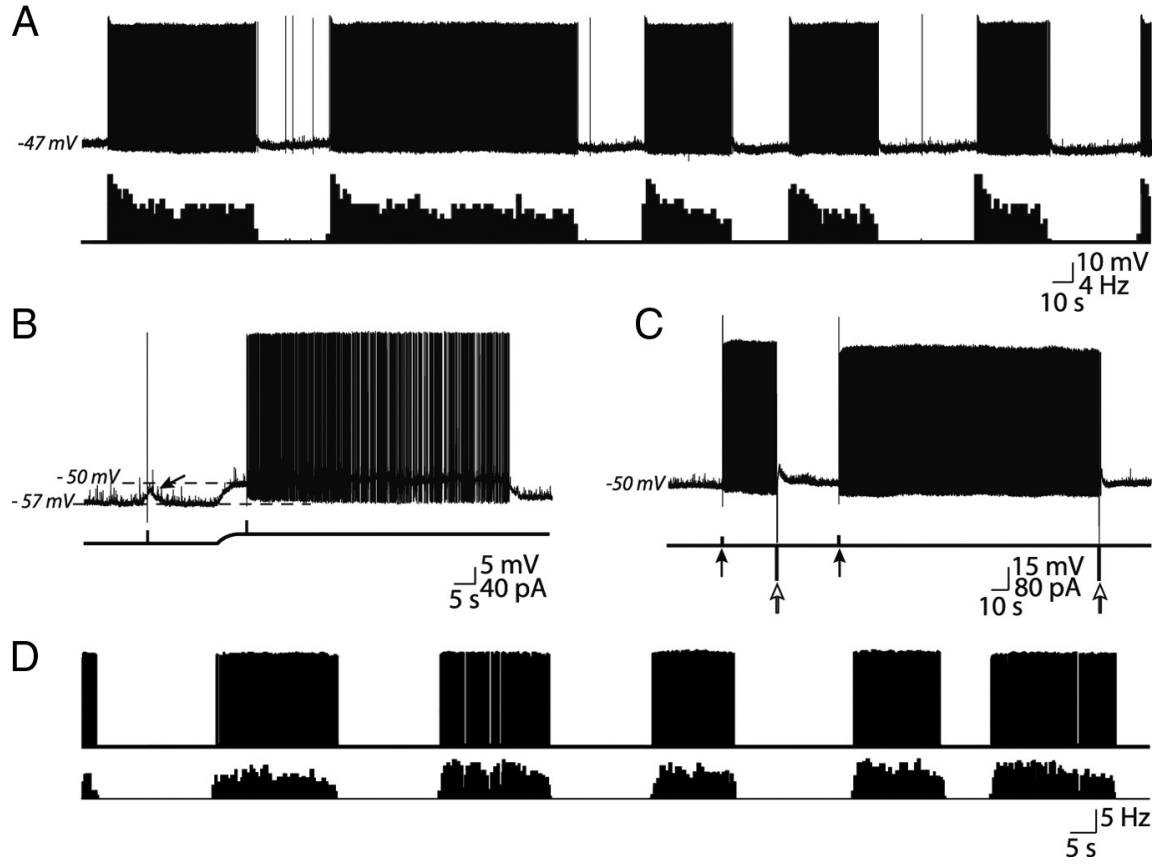

Figure 3. Electrical activity of VP neurons in acute hypothalamic slices and in vivo. A, A typical phasic activity recorded in a VP neuron from an acute slice, characterized by sequences of firing and silent periods (top trace). The bottom trace is the corresponding sequential stimulus histogram. $\boldsymbol{B}$, Example of a silent neuron in an acute slice at resting potential ( $-57 \mathrm{mV})$. Stimulation with a positive current pulse (bottom trace; black arrowhead) triggered a DAP (arrow). This DAP was not sufficient to trigger a plateau to support sustained firing. When the neuron was depolarized to $-50 \mathrm{mV}$ by injection of a sustained positive current, an identical stimulation induced firing (APs are truncated). Note that the activity ceased spontaneously despite sustained depolarization. C, Injection of brief positive currents (100 ms, $40 \mathrm{pA}$; black arrowheads) in a silent cell at resting potential ( $-50 \mathrm{mV}$ ) triggered a sustained firing activity, which was interrupted by passing a negative current (400 ms, $-120 \mathrm{pA}$; empty arrowheads). D, An example of electrical activity in a VP neuron recorded extracellularly in vivo (top trace). The bottom trace is the corresponding sequential distribution histogram of APs.

a Flaming/Brown P87 puller (Sutter Instrument) and filled with $1 \mathrm{~m}$ potassium acetate with $1 \%$ biocytin (Sigma-Aldrich). Electrode resistance varied from 150 to $250 \mathrm{M} \Omega$. Intracellular potentials from neurons were recorded through a single microelectrode using an Axoclamp-2A (Molecular Devices), which also permitted injection of currents.

Acute slice preparation. Wistar rats were anesthetized with isoflurane (5\% isoflurane- $95 \% \mathrm{O}_{2}$ ) and killed by decapitation using a guillotine. Immediately after opening the skull, the brain was removed and immersed in ice-cold oxygenated $\left(95 \% \mathrm{O}_{2}-5 \% \mathrm{CO}_{2}\right)$ medium for $1 \mathrm{~min}$. The composition of the perfusion medium was as follows (in $\mathrm{mM}$ ): 125 $\mathrm{NaCl}, 3 \mathrm{KCl}, 1.24 \mathrm{MgSO}_{4}, 1.3 \mathrm{KH}_{2} \mathrm{PO}_{4}, 25 \mathrm{NaHCO}_{3}, 2 \mathrm{CaCl}_{2}$, and 11 glucose. The brain was cut into a small cube that included the SON. The block was then fixed with cyanoacrylic glue on the top of the holder of a vibroslicer (MacIlwain; Campden Instruments). Three coronal sections $(400 \mu \mathrm{m})$ were made and transferred onto a filter paper (optic lens neutral cleaner) in contact with a ramp-style interface recording chamber (Israel and Poulain, 2000). The medium was perifused using a peristaltic pump (Gibson) at a rate of $1 \mathrm{ml} \cdot \mathrm{min}^{-1}$ and at constant temperature $\left(32 \pm 0.5^{\circ} \mathrm{C}\right)$. The slices were constantly oxygenated with humidified $95 \% \mathrm{O}_{2}-5 \% \mathrm{CO}_{2}$. The electrophysiological session began after a $1.5 \mathrm{~h}$ equilibration period. The microelectrode was positioned into the SON with a micromanipulator (Microcontrôle) under visual control using a dissecting microscope.

Comparison of phasic activity in slices obtained from adult female (240-260 g), lactating female (7-20 d of lactation), and adult male (250$280 \mathrm{~g})$ rats did not yield any differences in intraburst frequency (IF), burst duration (BD), and silent duration (SD) (supplemental Fig. S1, available at www.jneurosci.org as supplemental material). We thus performed our experiments on adult female rats.

In vivo experiments. Experiments were performed on lactating female Wistar rats (280-350 g) using a conventional procedure for recording extracellular action potentials in the SON (Poulain et al., 1977). Briefly, animals were first lightly anesthetized with isoflurane $\left(5 \%\right.$ isoflurane- $\left.95 \% \mathrm{O}_{2}\right)$, and then deeply anesthetized with urethane (1.3 $\mathrm{g} \cdot \mathrm{kg}^{-1}$ ) and set in a stereotaxic frame (Narishige). The surface of the brain was exposed and the sagittal sinus ligatured; a bipolar concentric stimulating electrode (Rhodes Medical Instruments) was then introduced via a dorsal approach into the pituitary stalk for antidromic identification of neurosecretory cells. Extracellular recordings of APs were obtained with glass micropipettes connected to a conventional electrophysiological apparatus as described previously (Poulain et al., 1977). Cells were tested during suckling and considered to be vasopressinergic when they did not react to suckling, contrary to the other cells, classified as oxytocinergic. The characteristics of the electrical activity were analyzed off-line from tape recordings.

Analyses of electrical activity. Electrical signals were visualized on a digital oscilloscope (Tektronix TDS 2012B), recorded directly on a recorder (Gould thermal array recorder TA 11; Gould), and stored on a hard disk through pClamp 9 software (Molecular Devices) through a Digidata 1300 interface (Molecular Devices). Stored electrical activity was replayed, and sequential frequency histograms were calculated over $1 \mathrm{~s}$ integration periods and plotted versus time using an appropriate program from pClamp9. Values are expressed as means $\pm \mathrm{SD}$. Data were analyzed using with the nonparametric Kruskal-Wallis test. Cumulative frequency or amplitude distributions of glutamatergic and GABAergic synaptic potentials were obtained from off-line analysis of unitary potentials using Axograph (Molecular Devices). Data were compared statistically with the paired Student test.

Identification of recorded neurons in vitro. To identify the phenotype of recorded cells in acute slice preparations and organotypic slice cultures, we used a method described in detail in our previous studies (Jourdain et al., 1996, 1999). The recording electrodes contained 1\% biocytin (SigmaAldrich). At the end of the intracellular recording session, neurons were filled with biocytin using hyperpolarizing current pulses $(-0.5 \mathrm{nA}, 0.4 \mathrm{~s}$, $2 \mathrm{~Hz}, 10-20 \mathrm{~min}$ ). Slices were then fixed in $4 \%$ paraformaldehyde and $0.15 \%$ picric acid for $2 \mathrm{~h}$ at room temperature and rinsed in $4 \%$ paraformaldehyde (two times; 20 min each time). Biocytin was visualized with streptavidin-conjugated Texas Red fluorescence (Biosys; diluted 1:400) with appropriate filters (Leitz DMR microscope; Leica). Slices then underwent double immunofluorescence using a mixture of primary antibodies, one being a monoclonal mouse Ig raised against oxytocin (OT)-related neurophysin [offered by H. Gainer, University of Toledo, Toledo, $\mathrm{OH}$, and characterized in the study by Ben-Barak et al. (1985)], and the other, a polyclonal rabbit serum raised against VP-associated neurophysin [offered by A. Robinson, David Geffen School of Medicine at UCLA, Los Angeles, CA, and characterized in the study by Roberts et al. (1991)]. After mounting with Fluoromount (Vectashield; Vector Laboratories), preparations were examined with epifluorescence with appropriate filters.

\section{Results}

\section{Basal electrical properties of VP neurons in organotypic} slice cultures

The electrical properties of 147 VP neurons were studied in hypothalamic cultures from 9-d-old rats after 5-10 weeks in vitro. A majority of VP neurons (67\%) displayed a slow irregular or continuous firing pattern, varying from 0.3 to $12 \mathrm{~Hz}(4.2 \pm 3.6 \mathrm{~Hz}$; $n=12$ ) (Fig. 2A1). Briefly, they had a mean resting membrane 


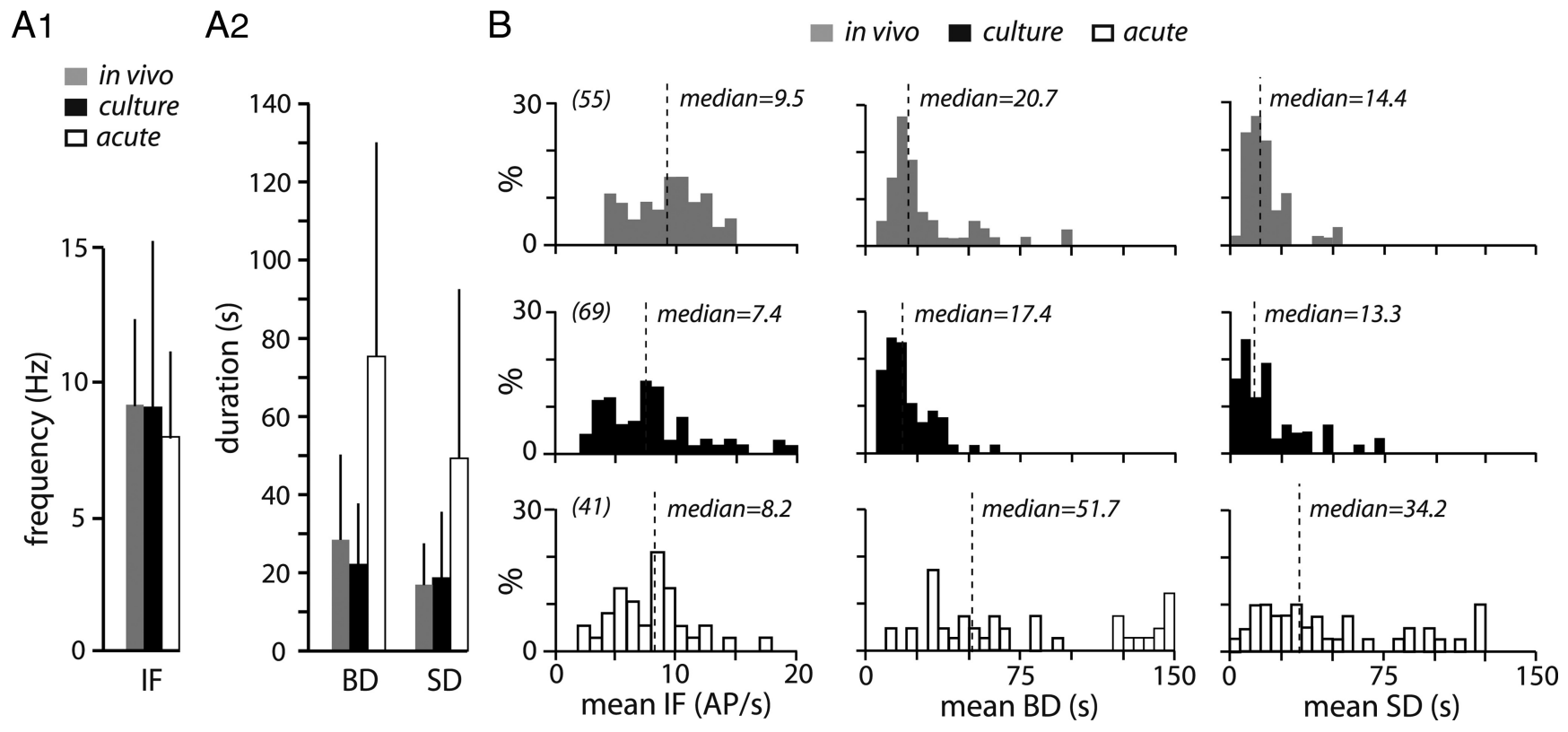

Figure 4. Comparison of phasic activity in vitro and in vivo. A1, Summary histogram showing the mean IF recorded extracellularly in vivo (gray columns; $n=55$ ) or intracellularly in organotypic slice cultures (black columns; $n=69$ ) and acute slices (empty columns; $n=41$ ). Results are expressed as mean \pm SD. $A 2$, Summary histogram recapitulating the mean BD and the mean SD for phasic activity in the three preparations. $\boldsymbol{B}$, Statistical distribution of mean IF, BD, and SD in the three groups of neurons. The vertical dotted line in each histogram represents the median value.

potential of $-64.7 \pm 3.6 \mathrm{mV}(n=20)$. They displayed APs with a mean amplitude of $59.8 \pm 8.9 \mathrm{mV}$ and a mean duration of $1.98 \pm 0.47 \mathrm{~ms}$ (means from 310 APs recorded from 10 cells). In addition to APs, a sustained synaptic activity was clearly visible (Fig. 2A2). In addition, the majority of VP neurons displayed endogenous properties typical of magnocellular neurons recorded in acute slices of rat hypothalamus and explants, such as a hyperpolarizing afterpotential after single spikes, an afterhyperpolarization (AHP) after bursts of APs (Fig. 2C, arrow), a frequency-dependent broadening of APs during a train of depolarizing pulses (Fig. $2 B 1, B 2)$, and the notch delaying the occurrence of firing at hyperpolarized potentials (Fig. $2 C$, arrowhead). One-third of VP cells displayed a DAP (see Fig. 7A2,C2,E) after a single or a few spikes.

Forty-seven VP neurons (36\% of total) spontaneously displayed a phasic behavior characterized by alternative bursts of APs and periods of silence (Fig. 2D). BD varied considerably from one cell to another (mean duration, $22.1 \pm 15.7 \mathrm{~s})(n=352$ from 69 neurons) (Fig. $2 D 1, D 2)$ and for a single cell during the recording session. The mean burst amplitude was $165 \pm 243$ APs ( $n=250$ from 34 neurons) and the mean IF also varied from cell to cell $(9.1 \pm 6.3 \mathrm{~Hz} ; n=69)$. As previously described in vivo (Poulain and Wakerley, 1982), the shape of bursts displayed a great variability, with a mean peak frequency of $19.4 \pm 11.0 \mathrm{~Hz}$. Silent periods (SD) also varied markedly from cell to cell, from 2 to $75 \mathrm{~s}$ (mean, $18.7 \pm 17.1 \mathrm{~s} ; n=69)$ (Fig. $2 D$ ) and, to a lesser extent, between successive bursts in a single neuron. As was reported in vivo (Poulain and Wakerley, 1982), some neurons displayed a few isolated APs during silent periods.

A few VP neurons (3\%) displayed a mixed activity consisting of a phasic activity interrupted by intermittent brief (5-9 s) highfrequency $(20-60 \mathrm{~Hz})$ discharges of APs, typical of oxytocinergic neurons (Jourdain et al., 1998), an activity reminiscent of that previously described in vivo (Moos and Ingram, 1995).

\section{Comparison of phasic activity in vitro and in vivo}

To compare the rhythmic behavior displayed by VP neurons in organotypic slice cultures with that described previously, we re- corded and analyzed such activity in acute slices of adult hypothalamus and in adult rats in vivo. Seventy-eight identified VP neurons were recorded from acute hypothalamic slices, among which $53 \%(n=41)$ displayed a phasic firing pattern (Fig. $3 A)$. In these cells, DAPs were able to trigger bursting activity when the membrane potential was sufficiently depolarized (Fig. $3 B, C$ ). Furthermore, injection of a brief negative current interrupted the bursts (Fig. 3C). These observations are similar to those reported in previous studies that first described the mechanism underlying this rhythmic behavior (Andrew and Dudek, 1984). In vivo, 55 neurons identified as vasopressinergic displayed a phasic activity (Fig. 3D). Their characteristics were as follows: IF, $9.2 \pm 3.0 \mathrm{~Hz}$; BD, $28.7 \pm 22.0 \mathrm{~s}$; and SD, $17.0 \pm 10.8 \mathrm{~s}$ (Fig. $4 A$ ), values equivalent to those reported previously [IF, $4-14 \mathrm{~Hz}$; BD, $10-25 \mathrm{~s}$; SD, 5-20 s in the study by Poulain et al. (1988)]. We next used these parameters to compare phasic activity among the three experimental models.

We found that IF was not statistically different (Kruskal-Wallis test, $p>0.05)$ in the three preparations $(9.1 \pm 6.3 \mathrm{~Hz}, n=69$, and $8.0 \pm 3.2 \mathrm{~Hz}, n=41$, for organotypic and acute slices, respectively) (Fig. 4A1). BD and SD, however, differed statistically between organotypic and acute slices. Values for organotypic cultures were similar $(p>0.05)$ to those measured in vivo (see above), whereas they were significantly increased $(p<0.05)$ in acute slices (75.4 \pm 55.4 and $49.3 \pm 43.9 \mathrm{~s} ; n=41)$ (Fig. 4 A2). These parameters characterizing phasic activity are summarized in Figure $4 B$ for the three preparations. The analysis revealed that the rhythmic firing pattern in VP neurons recorded from organotypic slice cultures is closely similar to the phasic activity recorded in vivo, whereas it differed markedly from that recorded in acute hypothalamic slices.

Phasic activity is regulated by extracellular osmolarity It has been shown previously that acute hyperosmotic stimulation triggers or accelerates phasic activity in vivo (Brimble and Dyball, 1977; Bourque and Renaud, 1984) and depolarizes VP neurons in vitro (Bourque, 1989; Oliet and Bourque, 1992, 


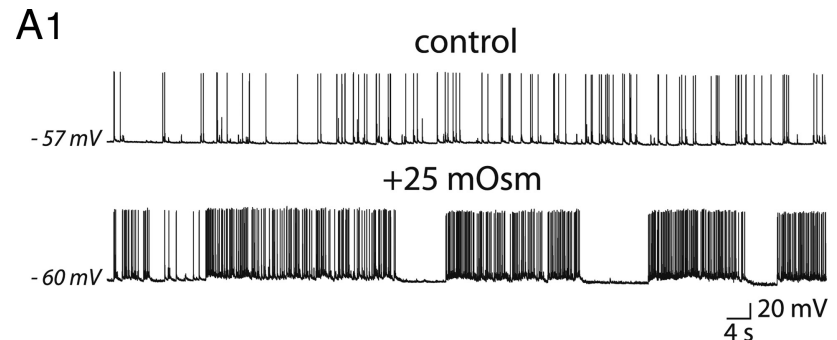

B1

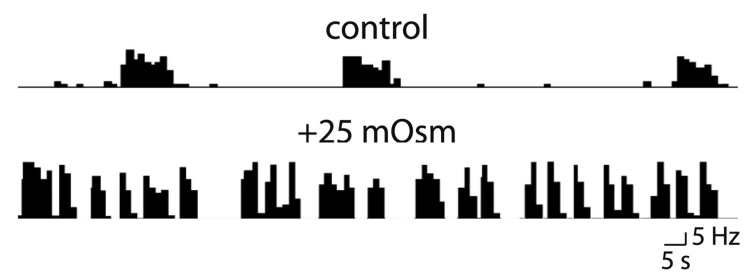

C1

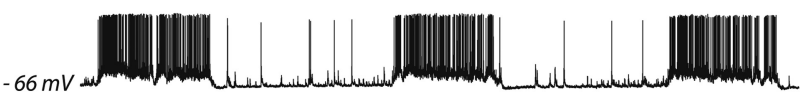

$-20 \mathrm{mOsm}(3 \mathrm{~min})$
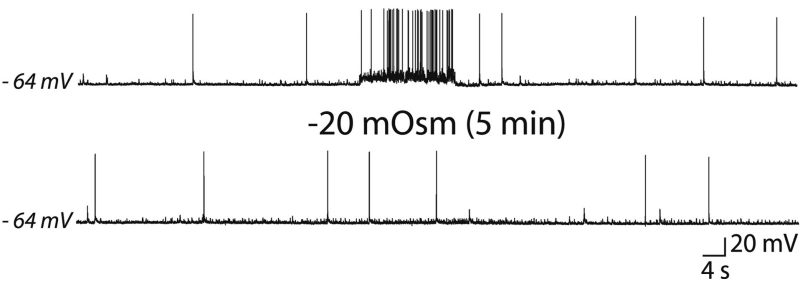

Figure 5. Phasic activity in VP neurons in organotypic slice cultures is regulated by extracellular osmolarity. $\boldsymbol{A} \mathbf{1}$, The spontaneous slow irregular activity in a VP neuron (top trace) progressively accelerated after application of a hyperosmotic stimulus $(+25$ $\mathrm{m} 0 \mathrm{sm})$; the cell finally displayed a phasic activity (bottom trace) $(\boldsymbol{A} 2 ; n=6) . \boldsymbol{B} 1$, Example of a neuron displaying a spontaneous phasic activity (sequential distribution histogram of APs) maintained under normal iso-osmotic (top trace, control) or hyperosmotic conditions (bottom trace, $+25 \mathrm{m0sm}$ ). B2, Summary histogram showing the value of the activity quotient $Q$ before (empty column) and after (black columns) application of the hyperosmotic stimulus (triangle). The osmotic stimulus dramatically increased $Q$, which progressively returned to its initial value during washing out (number of experiments is indicated in brackets). C1, Spontaneous phasic activity recorded in a VP neuron (top trace). A hypo-0smotic stress ( $-20 \mathrm{~m} 0 \mathrm{sm}$ ) progressively decreased activity (medium trace), thereby unmasking synaptic activity during a burst; this ultimately arrested (bottom trace) firing. C2, Summary histogram illustrating the effect of incubation in $-20 \mathrm{~m} 0 \mathrm{sm}$ medium $(n=4)$ on mean firing frequency.

1993a,b). Conversely, hypotonic media hyperpolarize magnocellular neurons (Oliet and Bourque, 1993a,b). This prompted us to test the effect of hypertonic (320 mOsm) and hypotonic (275 mOsm) stimuli on VP neurons in organotypic slice cultures. The hyperosmotic challenge rapidly (10-20 s) and progressively increased AP frequency in neurons firing at a low frequency $(0.1-4$ $\mathrm{Hz} ; n=7$ ) before triggering phasic activity in six of seven cells (Fig. 5A). The effect was totally reversible after washing. Hyperosmotic stimulation was also tested on 13 neurons displaying spontaneous phasic activity. In four cells, it caused an increase in $\mathrm{BD}(+88 \pm 31 \% ; p<0.05)$ without significantly changing SD, so that the $Q$ coefficient (the proportion of bursting time spent by a phasic cell during a fixed time) (Wakerley et al., 1975) increased from $0.19 \pm 0.03$ to $0.41 \pm 0.05(p<0.05)$. In six cells, hyperosmolarity reduced both $\mathrm{BD}$ and $\mathrm{SD}$ (Fig. $5 \mathrm{B1}$ ), consequently increasing $Q$ from $0.26 \pm 0.12$ to $0.81 \pm 0.26$ (Fig. 5B2). Concomitantly, we noticed a slight but significant increase in IF $(127 \pm 16 \% ; p<0.05)$ and in peak frequency $(119 \pm 10 \% ; p<$ $0.05)$. Finally, in the remaining three phasic neurons, hyperosmolarity depolarized membrane potential $(9.3 \pm 2.7 \mathrm{mV})$ accompanied by an increase in membrane conductance, leading to a sustained firing that terminated the rhythmic activity (data not shown).
A2

Reducing the osmolarity to $275 \mathrm{mOsm}$ had an opposite action on electrical activity since it inhibited firing in VP neurons, turning the phasic pattern into a slow irregular AP discharge, decreasing spike frequency by $86 \pm 7 \%(n=4)$ (Fig. $5 C 1, C 2)$. This effect was also completely reversible when the osmolarity was returned to $295 \mathrm{mOsm}$ (Fig. 5C2).

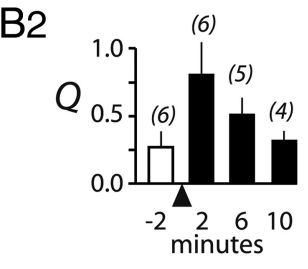

Control of phasic activity by $\kappa$-opiates The $\kappa$-opioid peptide dynorphin is synthesized and copackaged with VP in the same neurosecretory granules (Watson et al., 1982; Shuster et al., 2000). In vivo and in vitro studies established that activation of $\kappa$-opioid receptors on VP neurons inhibited phasic behavior (Brown et al., 1998) through the inhibition of DAP (Brown and Bourque, 2004). Conversely, antagonizing $\kappa$-opioid receptors was shown to facilitate phasic firing, revealing the key role played by endogenous dynorphin in regulating rhythmic activity (Brown and Bourque, 2004). We thus tested whether dynorphin would have a similar effect on the phasic activity displayed by VP neurons in organotypic slice cultures. We first found that dynorphin $(1 \mu \mathrm{M})$ decreased the amplitude of DAP from $4.7 \pm 0.8$ to $2.9 \pm 0.5 \mathrm{mV}[n=3$; $p<0.05$, a value similar to that reported in explants (Brown and Bourque, 2004)]. As expected, in six VP cells, dynorphin reduced phasic activity, decreasing $Q$ from $0.57 \pm 0.19$ to $0.13 \pm 0.07$. In five other VP cells, dynorphin switched the activity from phasic to a slow irregular firing (Fig. $6 A$ ), decreasing spike discharge by $75.4 \pm 12.7 \%$ of control values. In the three remaining VP neurons, dynorphin did not have any effect on firing activity.

We then applied the specific $\kappa$-opioid receptor antagonist BNI on phasically firing neurons to assess the role of endogenous $\kappa$-opiates on phasic activity. As clearly shown in Figure $6 B$, BNI $(1 \mu \mathrm{M})$ reduced SD and dramatically increased $\mathrm{BD}$, therefore increasing $Q$ from $0.35 \pm 0.10$ to $0.78 \pm 0.18(n=4 ; p<$ $0.05)$. BNI also increased IF (+101 $\pm 47.1 \% ; n=4 ; p<0.05)$. Together, the results obtained with the agonist and antagonist of $\kappa$-opiate receptors indicate that phasic activity in organotypic slice cultures is regulated by the endogenous release of dynorphin, as is the case in hypothalamic explants (Brown and Bourque, 2004; Brown et al., 2006) and in vivo (Brown et al., 1998, 2006).

The effects of extracellular osmolarity and $\kappa$-opiates on glutamatergic transmission were further analyzed in a TTX $(1 \mu \mathrm{M})$ containing medium. A hypertonic $(+25 \mathrm{mOsm})$ medium did not modify the frequency of miniature EPSPs (mEPSPs) $(+4.3 \pm$ $13.6 \% ; n=5 ; p>0.05$ ) (supplemental Fig. S3A1-A3, available at www.jneurosci.org as supplemental material) but unexpectedly slightly increased their amplitude $(+5.2 \pm 2.9 \% ; n=5 ; p<0.05)$ (supplemental Fig. S3A2,A3, available at www.jneurosci.org as supplemental material). Dynorphin and BNI decreased $(-30.9 \pm$ $11.7 \% ; n=4 ; p<0.05$ ) (supplemental Fig. S3B1-B3, available at www.jneurosci.org as supplemental material) and increased $(+25.7 \pm 9.8 \% ; n=7 ; p<0.05)$ (supplemental Fig. S3C1-C3, 
available at www.jneurosci.org as supplemental material) the frequency of $\mathrm{mEP}$ SPs, respectively, without alteration of mEPSP amplitude $(-2.5 \pm 4.1 \%, n=4$, $p>0.05$; and $+1.3 \pm 4.1 \%, n=7, p>$ 0.05 , respectively) (supplemental Fig. $\mathrm{S} 3 B, C$, available at www.jneurosci.org as supplemental material).

\section{DAP and phasic activity}

DAP was shown to play a key role in the phasic activity displayed by VP neurons in hypothalamic acute slices (Andrew and Dudek, 1983, 1984) and explants (GhamariLangroudi and Bourque, 1998). Furthermore, the regulation of phasic activity by $\kappa$-opiate receptors was seen to be mediated via a modulation of DAP (Brown and Bourque, 2004). It was thus of paramount importance to assess the presence and the role of DAP in the phasic activity of VP neurons in organotypic slice cultures. Among VP neurons displaying a spontaneous phasic activity, only 18 of 46 neurons (39\%) expressed a DAP after the induction of a single or a couple of APs (Fig. 7A1,A2,C1,C2,E). This suggested that DAPs were not necessarily involved in phasic activity (Fig. $7 B 1, B 2$ ). We therefore tested the effect of FFA, a blocker of cationic currents previously shown to inhibit DAP in explants (Ghamari-Langroudi and Bourque, 2002). In phasically active VP neurons displaying DAPs, FFA completely blocked DAPs, as expected, but it did not significantly alter the phasic pattern (Fig. 7C,D). FFA did not affect the rhythmic pattern of firing in phasically firing neurons that did not exhibit DAPs (Fig. 7D).

We next checked whether DAPs were able to trigger and maintain plateau-like potentials supporting AP firing in organotypic slice cultures. This role was first demonstrated in acute slices and explants with brief positive and negative current injections that were able to induce and terminate phasic activity, respectively (Andrew and Dudek, 1984; Armstrong et al., 1994; Li and Hatton, 1997; Ghamari-Langroudi and Bourque, 1998). We were able to reproduce such results in acute hypothalamic slices, as illustrated in Figure 3, $B$ and $C$. However, using identical protocols in organotypic slice cultures, neither positive nor negative currents were able to trigger or to stop bursts (Fig. $8 \mathrm{~A}$ ). Furthermore, in cultures treated with CNQX $(15 \mu \mathrm{M})$ to inhibit EPSPs, neither depolarizing membrane potential nor increasing the amplitude of the current pulse triggering DAPs was effective at generating sustained plateau potentials $(n=12)$ (Fig. $7 E)$.

\section{Role of glutamatergic input in phasic activity}

As has been suggested by several groups (Andrew and Dudek, 1984; Brown et al., 2004; Sabatier et al., 2004), synaptic activity may be an alternative process to generate phasic activity. Detailed analysis of the recordings obtained from cells in the organotypic slice cultures revealed that bursts were often preceded by an increased EPSP activity, which summated to form a plateau-like potential (Fig. 8B1-B3), independent of the presence of DAPs (Fig. 8 B2). On the contrary, it was obvious that the termination

\section{control}

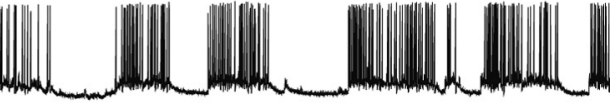

DYN (5 min)

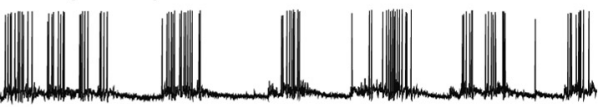

DYN (8 min)

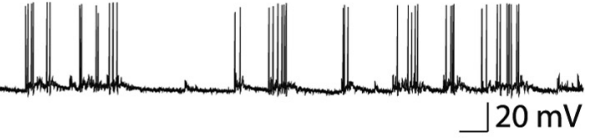

$5 \mathrm{~s}$ $20 \mathrm{mV}$

control

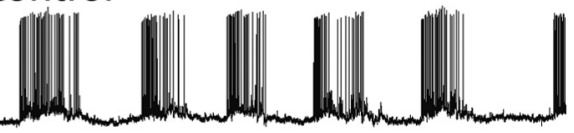

$\mathrm{BNI}$

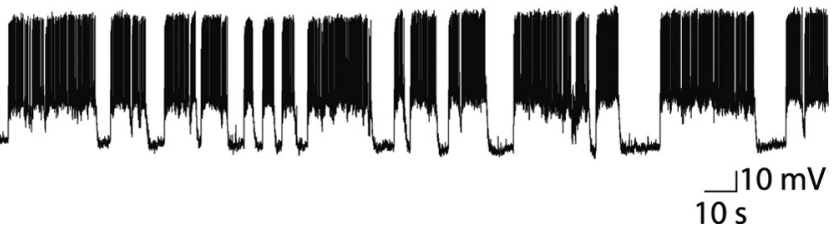

$10 \mathrm{~s}$

Figure 6. Phasic activity in VP neurons from organotypic slice cultures is controlled by $\kappa$-opiates. $\boldsymbol{A}$, Spontaneous phasic activity (control) was progressively altered by application of the opiate dynorphin (DYN) (1 $\mu \mathrm{m})$. After an 8 min treatment, the cell displayed a slow, irregular firing activity. $\boldsymbol{B}$, The specific $\boldsymbol{\kappa}$-receptor antagonist BNI dramatically accelerated phasic activity by simultaneously increasing the duration of the bursts and reducing the extent of the silent periods.

of the plateau was concomitant with a decrease of both the amplitude and the frequency of EPSPs (Fig. 8 B3). The role for synaptic activity was highlighted further when a slight hyperpolarization of membrane potential was imposed on the neurons, unmasking an abundant excitatory synaptic activity during the bursts (Fig. 8C1). Under such conditions, which permit detection and quantification of synaptic events before and during bursts (Fig. $8 C$ ), we noted that both EPSP frequency $(+177 \pm 75 \%$ of control values; $n=8)$ and amplitude $(+123 \pm$ $39 \% ; n=8$ ) were mostly increased during active periods (Fig. $8 D$ ). Confirmation of the key role played by glutamatergic activity in the regulation of phasic firing of VP neurons was obtained after application of the specific antagonists of AMPA-kainate and NMDA receptors, CNQX and D-AP-5 $(30 \mu \mathrm{M})$, respectively. In phasically firing VP neurons (Fig. 9A1), application of $15 \mu \mathrm{M}$ CNQX completely blocked AP firing (Fig. 9A2). Membrane depolarization restored AP discharge without generating phasic activity (Fig. 9A2) $(n=3)$. Similarly, application of a brief positive current failed to trigger a rhythmic behavior in CNQX-treated preparations (Fig. 9B2). The inhibitory effect of CNQX on phasic firing was dose dependent and tightly correlated to the inhibition of EPSP activity (supplemental Fig. S2, available at www. jneurosci.org as supplemental material), suggesting a link between glutamatergic transmission and VP neuron rhythmic behavior. In a different series of experiments, we saw that D-AP-5 switched the activity from phasic to a very slow irregular firing (Fig. 9C), decreasing AP frequency by $-87.5 \pm 8.7 \%(n=4)$.

Since phasic activity is regulated by external osmolarity and $\kappa$-opiate receptor activation, we analyzed effects on EPSP activity after modification of the external osmolarity or application of 

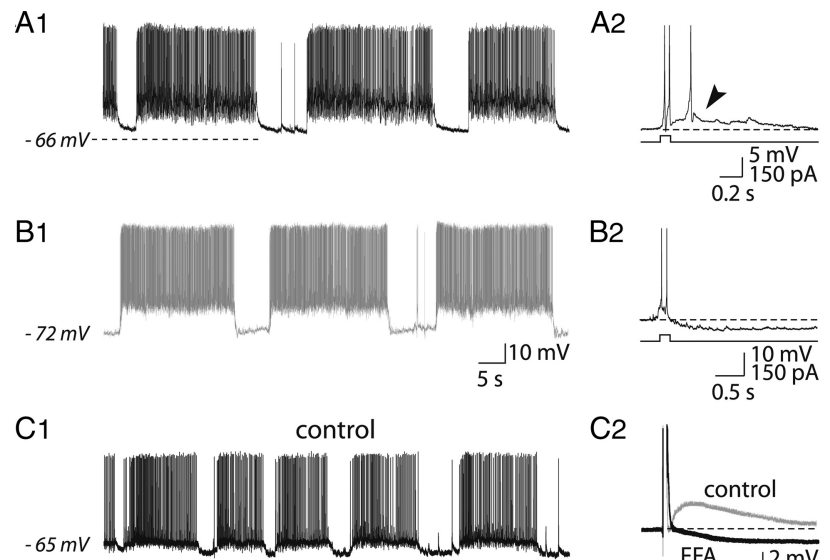

B2

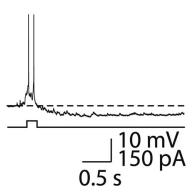

$\mathrm{C} 2$

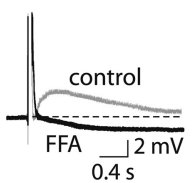

C3 FFA

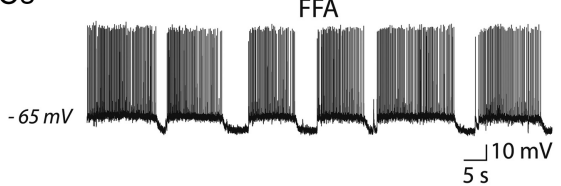

D DAP no DAP

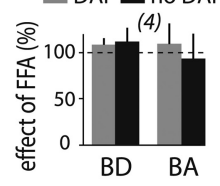

$\mathrm{E}$ in CNQX

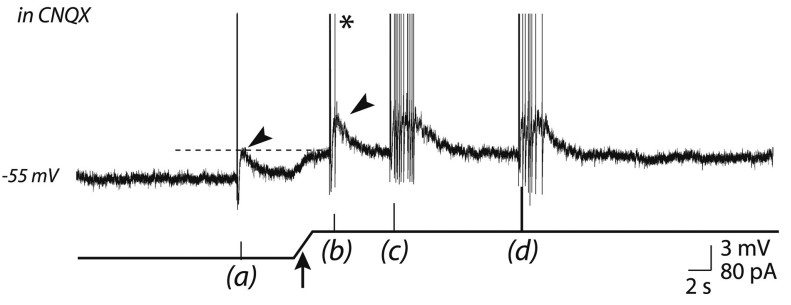

Figure 7. Phasic activity in VP neurons is not primarily linked to DAP. $\boldsymbol{A} 1, \boldsymbol{B} 1$, Spontaneous phasic activities recorded in two VP neurons. $A \mathbf{2}, \boldsymbol{B} 2$, In the presence of $C N Q X$, a positive current pulse (bottom trace) triggered APs, which were followed ( $\boldsymbol{A} \mathbf{2})$ or not (B2) by a marked DAP (A2, arrowhead). $C$, Spontaneous phasic activity $(\mathbf{C}$, control) recorded in a neuron in which APs triggered DAPs ( $\mathbf{2}$, in the presence of CNQX). C2, DAP was triggered by a burst of two APs (truncated) elicited by a positive current pulse ( $+20 \mathrm{pA}, 100 \mathrm{~ms}$ ). DAP was blocked by FFA ( 100 $\mu \mathrm{m})(\mathbf{C}$, black trace). ( 3 , Electrical activity recorded in the same neuron in the presence of FFA. $D$, Summary histogram showing that FFA did not significantly alter either the duration (BD) or amplitude (number of APs within a burst) (BA) of bursts in neurons that displayed (gray columns; $n=4$ ) or not (black columns; $n=4$ ) DAPs. $E$, CNQX abolished firing activity (resting potential, $-55 \mathrm{mV}$ ). Injection of a positive current pulse (bottom trace: $\boldsymbol{a},+60 \mathrm{pA}, 100 \mathrm{~ms}$ ) triggered a single AP that evoked a DAP (arrowhead). The cell was depolarized to the potential reached during the previous DAP ( $-52 \mathrm{mV}$; dashed line) by injection of a positive constant current ( $\boldsymbol{a}$, bottom trace, $+80 \mathrm{pA}$; arrow). An identical current pulse $(\boldsymbol{b})$ triggered two APs (APs were truncated). The resulting DAP (arrowhead) had a larger amplitude, sufficient to trigger a single AP (asterisk). Successive currents of larger amplitude $(\boldsymbol{c},+100 \mathrm{pA} ; \boldsymbol{d}, 130 \mathrm{pA})$ and duration $(\boldsymbol{d}, 300 \mathrm{~ms})$ triggered more robust DAPs, which triggered only very brief plateau potentials.

dynorphin. Hyperosmolarity increased significantly the frequency $(+90 \pm 49 \% ; p<0.05 ; n=7)$ and amplitude $(+43 \pm$ $24 \% ; p<0.05 ; n=7$ ) of glutamatergic events (Fig. 10A). Decreasing the osmolarity to $275 \mathrm{mOsm}$ reduced their frequency $(-49 \pm 10 \% ; n=4)$ and their amplitude $(-56 \pm 4 \% ; n=4)$ (Fig. 10B). In agreement with their respective action on phasic activity, BNI increased both the frequency $(+166 \pm 81 \% ; n=4$; $p<0.05)$ and amplitude $(+147 \pm 48 \% ; n=4)$ (Fig. 10C), whereas dynorphin decreased both the frequency $(-56.4 \pm$ $9.5 \% ; n=8 ; p<0.05)$ and the amplitude $(-58.8 \pm 10.2 ; n=8$; $p<0.05$ ) of EPSPs (Fig. 10D).

\section{Role of GABAergic inputs in phasic activity}

We examined the presence of $\mathrm{GABA}_{\mathrm{A}}$ receptor-induced IPSPs during silent periods and bursts (Fig. 11 A1,A2). Because the rest-
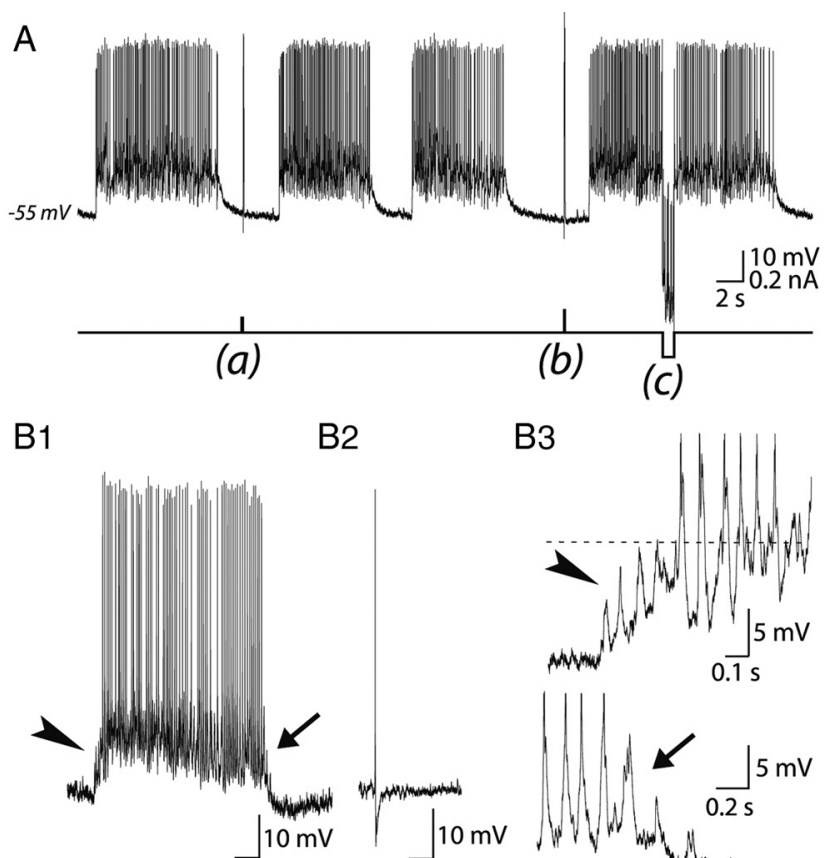

B2

B3
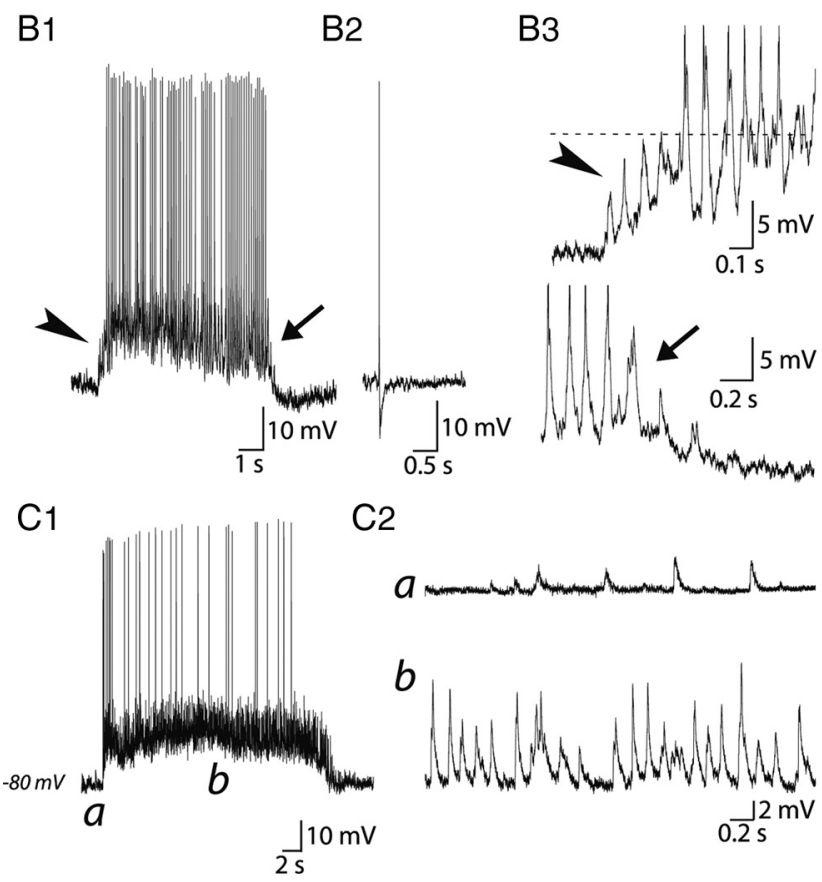

D1
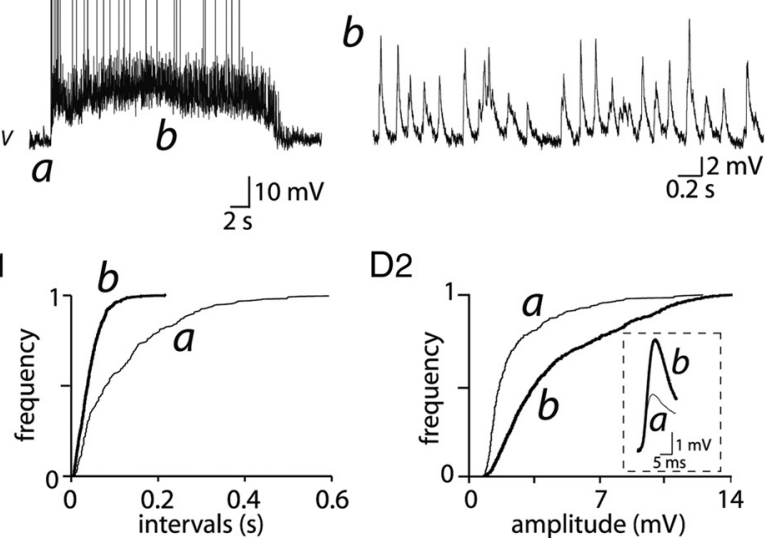

D2

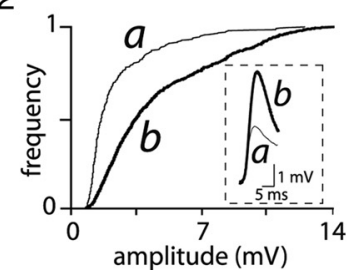

Figure 8. Glutamatergic afferents govern phasic activity in VP neurons. $A$, Example of a spontaneous phasic activity at resting membrane potential $(-55 \mathrm{mV})$ recorded from a VP neuron in an organotypic slice culture. Injection of positive currents (bottom trace, $\boldsymbol{a}$, $+0.15 \mathrm{nA}, 100 \mathrm{~ms} ; \boldsymbol{b},+0.2 \mathrm{nA}, 100 \mathrm{~ms}$ ) during silent periods did not trigger any burst. Conversely, application of a negative current (bottom trace, $c,-0.2 \mathrm{nA}, 1 \mathrm{~s}$ ) during a burst did not interrupt this burst. $\boldsymbol{B} 1$, A single burst from a recording obtained in a VP neuron displaying spontaneous phasic activity which did not express DAPS (B2). Higher magnification (B3) revealed that the beginning of the plateau ( $\boldsymbol{B}$ 1, arrowhead) consisted of the summation of EPSPs (top trace, arrowhead), which led to a plateau potential (dotted line; APs were truncated). On the contrary, the cessation of the plateau (B1, arrow) is attributable to the cessation of EPSPs (bottom trace, arrow). C1, Example of a burst recorded at a hyperpolarized membrane potential $(-80 \mathrm{mV})$. This unmasked a robust synaptic activity occurring during the burst. C2, Samples of electrical activity displayed at higher amplification showing EPSP activity during the silent period $(\boldsymbol{a})$ and within a burst $(\boldsymbol{b})$ of phasic activity. $\boldsymbol{D}$, Cumulative frequencies of EPSP intervals (D1) and amplitudes (D2) during a silent period ( $\boldsymbol{a}$, thin traces) and within a burst ( $\boldsymbol{b}$, thick traces). The inset represents the mean amplitude of EPSPs obtained from records in $\mathbf{C} \boldsymbol{a} \boldsymbol{a}$ (thin trace) and $\boldsymbol{C} \boldsymbol{b}$ (thick trace). 
ing membrane potential is close to the equilibrium potential for chloride ions (approximately $-70 \mathrm{mV}$ ) (Fig. 11A4), we had to depolarize the membrane to unmask IPSP activity during silent periods. This depolarization matches the membrane potential reached during the phasic plateau (Fig. 11A1). In all the neurons tested $(n=22)$, IPSP amplitude was dramatically increased $(+172 \pm 107 \%)$ during the bursts, whereas a rise in frequency was observed in only $64 \%$ of neurons ( $n=$ $14 ;+87 \pm 90 \% ; p<0.05$ ) (Fig. 11A2,A3).

Careful inspection of successive bursts during phasic activity of a single cell (Fig. $11 B 1$ ) showed that giant IPSPs could occur during the burst (Fig. 11B2), causing an inhibition of firing frequency. To study the impact of IPSPs on phasic activity, we compared within the same cells intraburst AP frequency as a function of membrane potential during bursts (Fig. 11C1). At depolarized potentials, AP firing rate should increase while, at the same time, IPSP amplitude will be augmented. Thus, the impact of GABAergic transmission on intraburst firing should be stronger. We therefore examined bursts in 20 neurones in which membrane potential was depolarized (from 10 to $15 \mathrm{mV}$ ) at one point during the recording session. Analyzing firing frequency versus membrane potential showed that depolarization increased IPSP amplitude (Fig. 11C1) while decreasing AP firing frequency $(-23 \pm 32 \% ; p<0.05)$ in 16 cells (Fig. 11C1,C2).

\section{Discussion}

We here investigated mechanisms involved in generating phasic activity in magnocellular VP neurons of the hypothalamus. Comparison of intraburst frequency, duration of bursts, and of silent periods clearly showed that the activity recorded in organotypic cultures was similar to the phasic activity displayed by VP neurons in vivo. Moreover, our recordings revealed that such activity took place in the absence of DAPs and was strongly dependent on glutamatergic synaptic activity. Together, our results highlight the importance of synaptic drive versus intrinsic membrane properties to account for the rhythmic behavior of magnocellular VP neurons.

\section{Phasic activity of VP neurons in vitro and in vivo}

VP neurons in organotypic slice cultures from postnatal hypothalamus possess passive and active electrical properties similar to those described in VP neurons in acute slices and explants from adult hypothalamus (Renaud and Bourque, 1991). Under normal conditions in the adult in vivo, only a fraction of VP neurons exhibits a phasic pattern of firing (Poulain and Wakerley, 1982). This also occurs in organotypic slice cultures in which a few VP neurons spontaneously displayed a phasic activity.

In our experiments, values for parameters like IF, BD, and SD in organotypic cultures (IF, 9.1 Hz; BD, $22 \mathrm{~s}$; SD, $18 \mathrm{~s}$ ) were similar to those in vivo (IF, $9.2 \mathrm{~Hz}$; BD, $29 \mathrm{~s} ; \mathrm{SD}, 17 \mathrm{~s}$ ), and in agreement with those reported in vivo (Poulain and Wakerley, 1982; Nissen et al., 1995; Moos et al., 1997; Gouzènes et al., 1998). In contrast, VP neurons in acute slices displayed longer bursts (75 s) and silent periods (49s). This has also been noted in previous studies of VP activity in acute slices [BD, $41 \mathrm{~s} ; \mathrm{SD}, 35 \mathrm{~s}$ in the study by Sabatier et al. (2004)] and in explants [BD, $47 \mathrm{~s}$; SD, $44 \mathrm{~s}$ in the study by Ghamari-Langroudi and Bourque (2000)]. Thus, the phasic activity of VP neurons in organotypic slice cultures appears more similar to that observed in vivo than in acute slices.

As already described in acute slices and in vivo (for review, see Brown and Bourque, 2006), the phasic activity expressed by VP neurons in organotypic cultures was facilitated and inhibited by hypertonic and hypotonic stimuli, respectively. As expected (Brown et al., 1998; Brown and Bourque, 2004), this activity was dependent on the endogenous release of dynorphin whose action on $\kappa$-opiate receptors regulated burst duration.

\section{Role of DAPs in phasic activity}

Studies in acute slice preparations (Andrew and Dudek, 1984) revealed an intrinsic property, described as DAP, that has been 


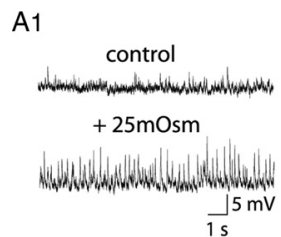

B1
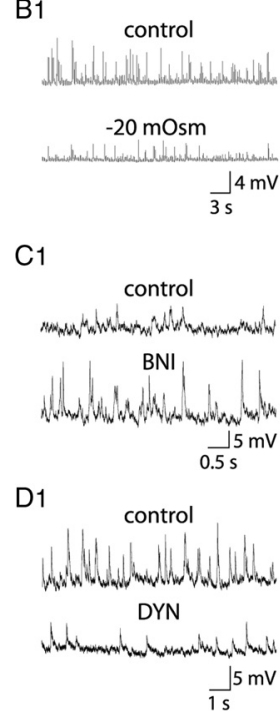

A2

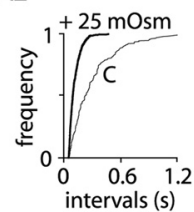

B2

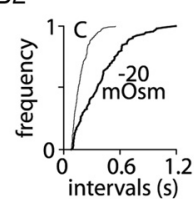

C2

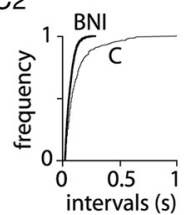

D2

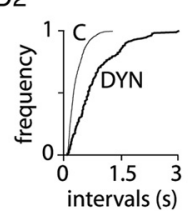

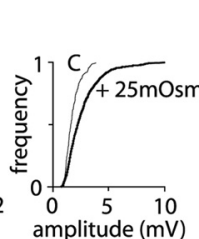
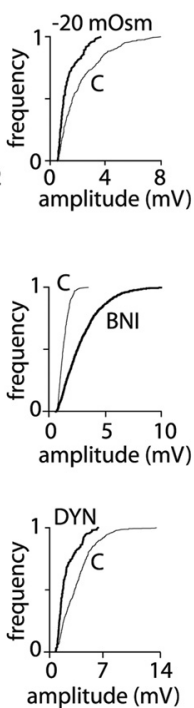

B3

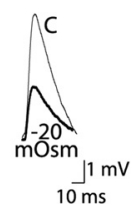

C3

A3

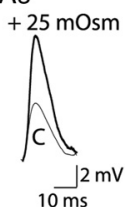

$2 \mathrm{mV}$

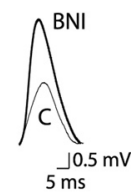

D3

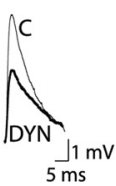

Figure 10. EPSPs in VP neurons are regulated by osmolarity and opiates. A1, B1, C1, D1, Samples of recordings of VP neurons in organotypic slice cultures under control conditions (top traces) or during stimulation by a hyperosmotic $(+25 \mathrm{~m} 0 \mathrm{sm})(\boldsymbol{A 1})$ or a hypo-osmotic medium ( $-20 \mathrm{m0sm}$; B1), application of BNI (C1) or dynorphin (DYN) (D1). A2, B2, C2, D2, Respective cumulative frequencies for EPSP intervals (left traces) and amplitudes (right traces) (C, control). $A 3, B 3, C 3, D 3$, Mean amplitude of EPSPs obtained during control (C) (thin traces), or hyperosmotic (A3, thick trace), hypo-osmotic (B3, thick trace) conditions, or application of BNI (C3, thick trace) or dynorphin (DYN) (D3, thick trace), respectively. Hyperosmotic stimulation and BNI application significantly increased both frequency and amplitude of EPSPs, whereas hypoosmotic stimulation and dynorphin significantly decreased EPSP amplitude and frequency.

considered essential for phasic activity. Furthermore, compounds affecting DAP amplitude, such as FFA (GhamariLangroudi and Bourque, 1998), dynorphin, and BNI (Brown and Bourque, 2004) alter phasic activity, which argues in favor of a tight relationship between the two processes.

In organotypic slice cultures, however, we could not establish such a link. Only $40 \%$ of phasically active cells displayed DAPs, and when DAPs were present, their suppression by FFA had no effect on phasic activity. In addition, we found that DAP per se was not sufficient to support phasic activity, as has been noted in recordings from isolated magnocellular neurons (Oliet and Bourque, 1992). Conversely, we reproduced the actions of dynorphin and BNI on phasic firing, but these effects were mediated through the modulation of excitatory synaptic activity. Our data therefore argue against an essential role for DAPs in the generation of phasic pattern in VP neurons. An alternative possibility, already proposed in previous reports (Andrew and Dudek, 1984; Nissen et al., 1994, 1995; Moos et al., 1997; Brown et al., 2004), is that such phasic activity is controlled by synaptic activity from glutamatergic inputs.

\section{Role of glutamatergic inputs in phasic activity}

Glutamate synapses represent $>20 \%$ of the synaptic input impinging on VP cells (El Majdoubi et al., 1996). In vivo experiments unambiguously showed that glutamate receptor blockers reversibly interrupted phasic activity in VP neurons (Nissen et al., 1994,

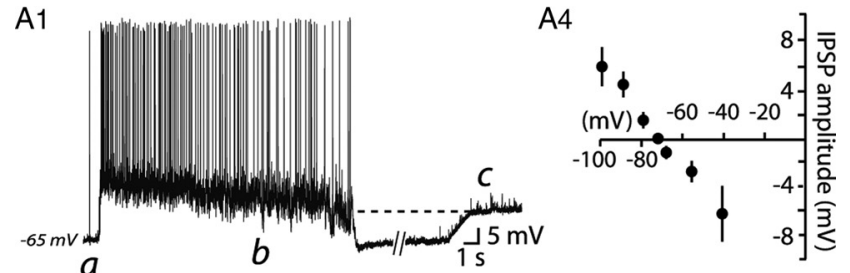

$\mathrm{A} 2$
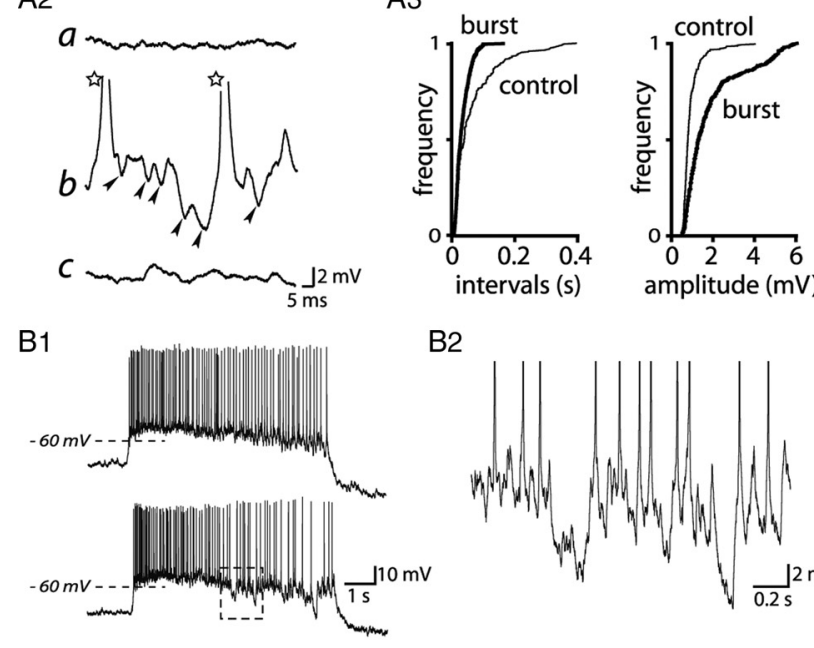

B2
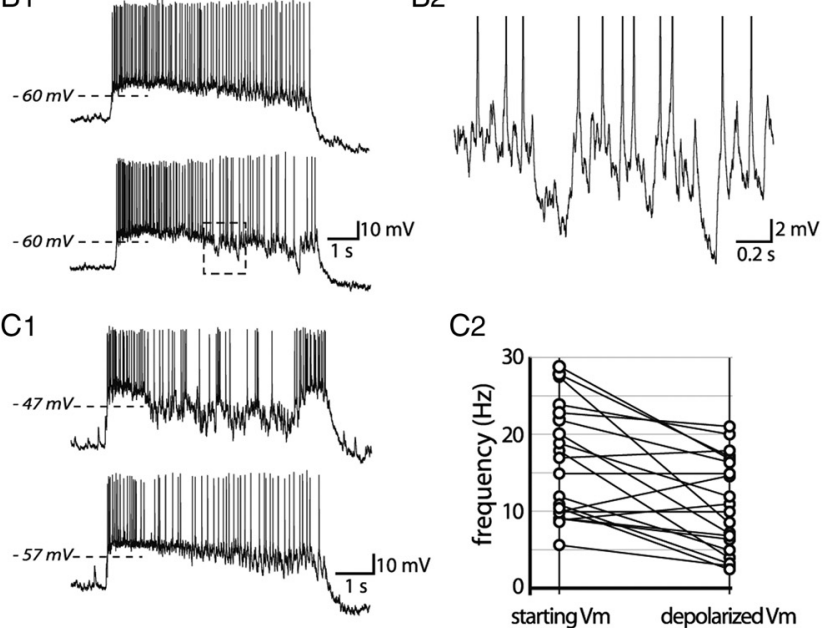

C2

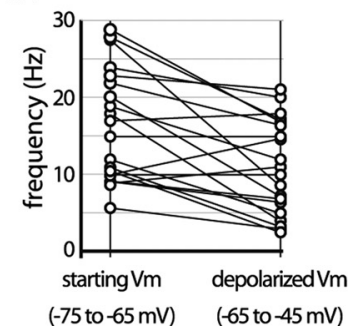

Figure 11. IPSP activity during bursts. A1, Left hand, Example of a burst of APs during phasic activity. At the end of the burst, a positive current was injected into the cell to bring the membrane potential close to the value reached during the plateau ( $\boldsymbol{c}$; dashed line). $\boldsymbol{A} \mathbf{2}$, Extracts of the trace shown in $\boldsymbol{A} \mathbf{1}$ at different periods $(\boldsymbol{a}-\boldsymbol{c})$. IPSPs were clearly detected within the burst $(\boldsymbol{b}$; arrowheads; stars indicate truncated APs). A3, Cumulative distribution for IPSP intervals and amplitude during silent (control; thin trace) and bursting (thick trace) periods. A4, Relationship between IPSP amplitude and membrane potential revealing a reversal potential for IPSPs near $-70 \mathrm{mV}$. B1, Example of two bursts recorded in the same neuron at the same membrane potential $(-75 \mathrm{mV})$. The dashed lines indicate the mean membrane potential during the plateau $(-60 \mathrm{mV})$. In the bottom trace, large hyperpolarizations occurred during the burst (dotted square), which are shown at higher magnification in B2. C1, Example of two bursts recorded in the same neuron that reached different average potentials during the plateau. $\mathbf{C}$, Mean frequency of APs (ordinates) within bursts recorded at starting membrane potential (starting Vm) and at a more depolarized potential (depolarized Vm), in 20 neurons.

1995; Moos et al., 1997), whereas glutamatergic receptor agonists triggered (Nissen et al., 1995) or accelerated (Moos et al., 1997) phasic activity. Glutamate was shown to be the sole source of EPSPs in coronal slices in vitro (van den Pol et al., 1990; Wuarin and Dudek, 1993; Yang et al., 1994; Richard and Bourque, 1995; Jourdain et al., 1998). In our cultures, blockade of EPSPs suppressed phasic activity even when firing was triggered and maintained with a depolarizing current indicating that glutamatergic EPSPs are required for generating rhythmic bursts. In addition, when AP firing was arrested by hyperpolarization, summation of bursts of synaptic potentials ultimately reached plateau-like depolarizations. These observations are similar to those of Yang et al. (1994) that showed that stimulation of the organum vasculosum 
lamina terminalis (OVLT) triggered a slow depolarizing envelop resulting from EPSP summation. Whereas glutamatergic activity appeared to be driving the phasic bursts, GABAergic transmission was also enhanced during these active periods, thereby attenuating AP firing rate. This could be an important process to prevent excessive firing discharge and the associated fatigue of vasopressin secretion (Ingram et al., 1982).

Exposure of the neurons to osmotic challenges modified both the frequency and amplitude of EPSPs, while affecting to a less extent mEPSP amplitude. These findings are in agreement with an action of osmotic stimuli on glutamatergic neurons impinging on VP cells, as previously described (Richard and Bourque, 1995). Therefore, in any case, blocking glutamatergic synaptic transmission with CNQX abolished the phasic firing triggered by a hyperosmotic stimulus (Fig. 9B1), as has been noted in explants of adult hypothalamic tissue (Richard and Bourque, 1995). This is in agreement with in vitro data indicating that the osmosensitive OVLT sends a glutamatergic synaptic input to SON neurons (Richard and Bourque, 1995; Inenaga et al., 1997). Although the distant OVLT is not included in our hypothalamic organotypic slice cultures, another osmosensitive area, the median preoptic nucleus (Bourque et al., 1994), closer to the SON, is probably present and could account for the osmotic modulation of EPSP activity in VP neurons.

In our study, in addition to a slight decrease in DAP amplitude (Brown and Bourque, 2004), we found that dynorphin dramatically reduced both glutamatergic EPSPs and phasic activity in VP neurons. In TTX-containing medium, we found that dynorphin decreased the frequency of mEPSPs, confirming its presynaptic action as demonstrated previously (Iremonger and Bains, 2009). Presynaptic modulation of glutamatergic input by dynorphin or its agonists has been noted in other structures, including the nucleus accumbens (Hjelmstad and Fields, 2003), hippocampus (Wagner et al., 1993), nucleus tractus solitarius (Rhim et al., 1993), and spinal cord (Randić et al., 1995).

The respective contribution of intrinsic properties like DAPs or osmosensitivity, and extrinsic factors like synaptic transmission in regulating phasic activity remains to be determined. DAPs appear to play a critical role in preparations in which synaptic afferents are severed from their soma. In acute slices of adult tissue, what remains is essentially miniature postsynaptic activity. Sabatier et al. (2004) proposed that the amplitude of DAPs, and thus their impact on phasic activity, is likely to be larger in acute slices than in vivo. It should be noted that in explants (Armstrong et al., 1994) and in acute slices (J.-M. Israel, unpublished results), not only do VP and OT neurons share basic intrinsic properties, but they both can display DAPs as well as phasic firing. In contrast, magnocellular neurons in vivo, as noted above, receive a strong glutamatergic synaptic input. In acute slices from immature animals, synaptic events were sensitive to TTX, indicating that local glutamatergic neurons do project on magnocellular neurons (Wuarin and Dudek, 1993). This is also the case in organotypic slice cultures originating from postnatal animals in which glutamatergic afferents are preserved (Jourdain et al., 1999).

Another intrinsic property likely to affect phasic activity is the osmosensitivity of the VP neurons themselves. Although isolated cells do not display phasic activity in the absence of synaptic input, hyperosmotic and hypo-osmotic media upregulate and downregulate, respectively, a mechanosensitive cationic conductance that modulates membrane potential (Oliet and Bourque, 1993a,b). In our organotypic cultures, maintaining cells in a hyperosmotic medium depolarized some VP neurons via an increase in membrane conductance. This could constitute, therefore, an additional mechanism allowing the cell to finely tune its response to afferent input.

\section{A local glutamatergic network supports phasic activity}

It has been established that most direct innervations of SON neurons derives from interneurons in the region adjacent to the nucleus (Léránth et al., 1975). Both anatomical (Tribollet and Dreifuss, 1981; Oldfield et al., 1992; Roland and Sawchenko, 1993; Csáki et al., 2002) and electrophysiological (Chaudhry et al., 1989; Wuarin and Dudek, 1993; Boudaba et al., 1997) observations in vivo clearly showed that local glutamatergic synaptic circuits exist in the hypothalamus. We showed that glutamatergic neurons survive in organotypic slice cultures from postnatal hypothalamus (Jourdain et al., 1999). Without excluding a role for extrahypothalamic glutamate afferents, for example from the OVLT, our observations highlight the importance of a local, intrahypothalamic pool of glutamatergic neurons driving the electrical activity of VP neurons. Whether the rhythmic pattern exhibited by glutamate afferents reflects an intrinsic property of glutamate neurons, or an emerging network behavior, is open to question. In vivo, it is well known that phasic activity between neighboring neurons is not synchronized, which implies that there is no synchronization of glutamate neurons when they are bursting, or that VP neurons receive input from different glutamate neurons exhibiting different patterns. This would be a major difference with the pool of glutamatergic neurons that govern the synchronized bursting activity of magnocellular OT neurons (Jourdain et al., 1998, 1999; Israel et al., 2003, 2008).

\section{References}

Andrew RD, Dudek FE (1983) Burst discharge in mammalian neuroendocrine cells involves an intrinsic regenerative mechanism. Science 221:1050-1052.

Andrew RD, Dudek FE (1984) Analysis of intracellularly recorded phasic bursting by mammalian neuroendocrine cells. J Neurophysiol 51:552-566.

Armstrong WE, Smith BN, Tian M (1994) Electrophysiological characteristics of immunochemically identified rat oxytocin and vasopressin neurones in vitro. J Physiol 475:115-128.

Ben-Barak Y, Russell JT, Whitnall MH, Ozato K, Gainer H (1985) Neurophysin in the hypothalamo-neurohypophysial system. I. Production and characterization of monoclonal antibodies. J Neurosci 5:81-97.

Boudaba C, Schrader LA, Tasker JG (1997) Physiological evidence for local excitatory synaptic circuits in the rat hypothalamus. J Neurophysiol 77:3396-3400.

Bourque CW (1989) Ionic basis for the intrinsic activation of rat supraoptic neurones by hyperosmotic stimuli. J Physiol 417:263-277.

Bourque CW, Renaud LP (1984) Activity patterns and osmosensitivity of rat supraoptic neurones in perfused hypothalamic explants. J Physiol 349:631-642.

Bourque CW, Oliet SH, Richard D (1994) Osmoreceptors, osmoreception, and osmoregulation. Front Neuroendocrinol 15:231-274.

Brimble MJ, Dyball RE (1977) Characterization of the responses of oxytocin- and vasopressin-secreting neurones in the supraoptic nucleus to osmotic stimulation. J Physiol 271:253-271.

Brown CH, Bourque CW (2004) Autocrine feedback inhibition of plateau potentials terminates phasic bursts in magnocellular neurosecretory cells of the rat supraoptic nucleus. J Physiol 557:949-960.

Brown CH, Bourque CW (2006) Mechanisms of rhythmogenesis: insights from hypothalamic vasopressin neurons. Trends Neurosci 29:108-115.

Brown CH, Ludwig M, Leng G (1998) $\kappa$-Opioid regulation of neuronal activity in the rat supraoptic nucleus in vivo. J Neurosci 18:9480-9488.

Brown CH, Bull PM, Bourque CW (2004) Phasic bursts in rat magnocellular neurosecretory cells are not intrinsically regenerative in vivo. Eur J Neurosci 19:2977-2983.

Brown CH, Leng G, Ludwig M, Bourque CW (2006) Endogenous activation 
of supraoptic nucleus kappa-opioid receptors terminates spontaneous phasic bursts in rat magnocellular neurosecretory cells. J Neurophysiol 95:3235-3244.

Brussaard AB, Kits KS, de Vlieger TA (1996) Postsynaptic mechanism of depression of GABAergic synapses by oxytocin in the supraoptic nucleus of immature rat. J Physiol 497:495-507.

Brussaard AB, Kits KS, Baker RE, Willems WP, Leyting-Vermeulen JW, Voorn P, Smit AB, Bicknell RJ, Herbison AE (1997) Plasticity in fast synaptic inhibition of adult oxytocin neurons caused by switch in GABAA receptor subunit expression. Neuron 19:1103-1114.

Cazalis M, Dayanithi G, Nordmann JJ (1985) The role of patterned burst and interburst interval on the excitation-coupling mechanism in the isolated rat neural lobe. J Physiol 369:45-60.

Chaudhry MA, Dyball RE, Honda K, Wright NC (1989) The role of interconnection between supraoptic nucleus and anterior third ventricular region in osmoregulation in the rat. J Physiol 410:123-135.

Csáki A, Kocsis K, Kiss J, Halász B (2002) Localization of putative glutamatergic/aspartatergic neurons projecting to the supraoptic nucleus area of the rat hypothalamus. Eur J Neurosci 16:55-68.

Dutton A, Dyball RE (1979) Phasic firing enhances vasopressin release from the rat neurohypophysis. J Physiol 290:433-440.

El Majdoubi M, Poulain DA, Theodosis DT (1996) The glutamatergic innervation of oxytocin- and vasopressin-secreting neurons in the rat supraoptic nucleus and its contribution to lactation-induced synaptic plasticity. Eur J Neurosci 8:1377-1389.

Ghamari-Langroudi M, Bourque CW (1998) Caesium blocks depolarizing after-potentials and phasic firing in rat supraoptic neurones. J Physiol 510:165-175.

Ghamari-Langroudi M, Bourque CW (2000) Excitatory role of the hyperpolarization-activated inward current in phasic and tonic firing of rat supraoptic neurons. J Neurosci 20:4855-4863.

Ghamari-Langroudi M, Bourque CW (2002) Flufenamic acid blocks depolarizing afterpotentials and phasic firing in rat supraoptic neurones. J Physiol 545:537-542.

Gouzènes L, Desarménien MG, Hussy N, Richard P, Moos FC (1998) Vasopressin regularizes the phasic firing pattern of rat hypothalamic magnocellular vasopressin neurones. J Neurosci 18:1879-1885.

Hatton GI (1982) Phasic bursting activity of rat paraventricular neurones in the absence of synaptic transmission. J Physiol 327:273-284.

Hjelmstad GO, Fields HL (2003) Kappa opioid receptor activation in the nucleus accumbens inhibits glutamate and GABA release through different mechanisms. J Neurophysiol 89:2389-2395.

Hussy N, Deleuze C, Pantaloni A, Desarménien MG, Moos F (1997) Agonist action of taurine on glycine receptors in rat supraoptic magnocellular neurones: possible role in osmoregulation. J Physiol 502:609-621.

Inenaga K, Cui L-N, Nagatomo T, Honda E, Ueta Y, Yamashita H (1997) Osmotic modulation in glutamatergic excitatory synaptic inputs to neurons in the supraoptic nucleus of rat hypothalamus in vitro. J Neuroendocrinol 9:63-68.

Ingram CD, Bicknell RJ, Brown D, Leng G (1982) Rapid fatigue of neuropeptide secretion during continual electrical stimulation. Neuroendocrinology 35:424-428.

Iremonger KJ, Bains JS (2009) Retrograde opioid signaling regulates glutamatergic transmission in the hypothalamus. J Neurosci 29:7349-7358.

Israel JM, Poulain DA (2000) $17 \beta$-Oestradiol modulates in vitro electrical properties and responses to kainate of oxytocin neurones in lactating rats. J Physiol 524:457-470.

Israel JM, Le Masson G, Theodosis DT, Poulain DA (2003) Glutamatergic input governs periodicity and synchronization of bursting activity in oxytocin neurons in hypothalamic organotypic cultures. Eur J Neurosci 17:2619-2629.

Israel JM, Poulain DA, Oliet SHR (2008) Oxytocin-induced postinhibitory rebound firing facilitates bursting activity in oxytocin neurons. J Neurosci 28:385-394.

Jourdain P, Poulain DA, Theodosis DT, Israel JM (1996) Electrical properties of oxytocin neurons in organotypic cultures from postnatal rat hypothalamus. J Neurophysiol 76:2772-2785.

Jourdain P, Israel JM, Dupouy B, Oliet SH, Allard M, Vitiello S, Theodosis DT, Poulain DA (1998) Evidence for a hypothalamic oxytocin-sensitive pattern-generating network governing oxytocin neurons in vitro. J Neurosci 18:6641-6649.
Jourdain P, Dupouy B, Bonhomme R, Poulain DA, Israel JM, Theodosis DT (1999) Visualization of local afferent inputs to magnocellular oxytocin neurons in vitro. Eur J Neurosci 11:1960-1972.

Kabashima N, Shibuya I, Ibrahim N, Ueta Y, Yamashita H (1997) Inhibition of spontaneous EPSCs and IPSCs by presynaptic GABAB receptors on rat supraoptic magnocellular neurons. J Physiol 504:113-126.

Kombian SB, Mouginot D, Hirasawa M, Pittman QJ (2000) Vasopressin preferentially depresses excitatory over inhibitory synaptic transmission in the rat supraoptic nucleus in vitro. J Neuroendocrinol 12:361-367.

Léránth C, Záborszky L, Marton J, Palkovits M (1975) Quantitative studies on the supraoptic nucleus in the rat. I. Synaptic organization. Exp Brain Res 22:509-523.

Li Z, Hatton GI (1997) $\mathrm{Ca}^{2+}$ release from internal stores: role in generating depolarizing after-potentials in rat supraoptic neurones. J Physiol 498:339-350.

Moos FC, Ingram CD (1995) Electrical recordings of magnocellular supraoptic and paraventricular neurons displaying both oxytocin- and vasopressinrelated activity. Brain Res 669:309-314.

Moos FC, Rossi K, Richard P (1997) Activation of N-methyl-D-aspartate receptors regulates basal electrical activity of oxytocin and vasopressin neurons in lactating rats. Neuroscience 77:993-1002.

Nissen R, Hu B, Renaud LP (1994) N-Methyl-D-aspartate receptors antagonist ketamine selectively attenuates spontaneous phasic activity of supraoptic vasopressin neurons in vivo. Neuroscience 59:115-120.

Nissen R, Hu B, Renaud LP (1995) Regulation of spontaneous phasic firing of rat supraoptic vasopressin neurones in vivo by glutamate receptors. J Physiol 484:415-424.

Oldfield BJ, Hards DK, McKinley MJ (1992) Neurons in the median preoptic nucleus of the rat with collateral branches to the subfornical organ and supraoptic nucleus. Brain Res 586:86-90.

Oliet SH, Bourque CW (1992) Properties of supraoptic magnocellular neurones isolated from the adult rat. J Physiol 455:291-306.

Oliet SH, Bourque CW (1993a) Mechanosensitive channels transduce osmosensitivity in supraoptic neurons. Nature 364:341-343.

Oliet SH, Bourque CW (1993b) Steady-state osmotic modulation of cationic conductance in neurons of rat supraoptic nucleus. Am J Physiol 265:R1475-R1479.

Poulain DA, Wakerley JB (1982) Electrophysiology of hypothalamic magnocellular neurones secreting oxytocin and vasopressin. Neuroscience 7:773-808.

Poulain DA, Wakerley JB, Dyball RE (1977) Electrophysiological differentiation of oxytocin- and vasopressin-secreting neurones. Proc R Soc Lond B Biol Sci 19:367-384.

Poulain DA, Brown D, Wakerley JB (1988) Statistical analysis of patterns of electrical activity in vasopressin and oxytocin-secreting neurones. In: Pulsatility in neuroendocrine systems (Leng G, ed), pp 119-154. Boca Raton, FL: CRC.

Randić M, Cheng G, Kojic L (1995) $\kappa$-Opioid receptor agonists modulate excitatory transmission in substantia gelatinosa neurons of the rat spinal cord. J Neurosci 15:6809-6826.

Renaud LP, Bourque CW (1991) Neurophysiology and neuropharmacology of hypothalamic magnocellular neurons secreting vasopressin and oxytocin. Prog Neurobiol 36:131-169.

Rhim H, Glaum SR, Miller RJ (1993) Selective opioid agonists modulate afferent transmission in the rat nucleus tractus solitarius. J Pharmacol Exp Ther 264:795-800.

Richard D, Bourque CW (1995) Synaptic control of rat supraoptic neurones during osmotic stimulation of the organum vasculosum lamina terminalis in vitro. J Physiol 489:567-577.

Roberts MM, Robinson AG, Hoffman GE, Fitzsimmons MD (1991) Vasopressin transport regulation is coupled to the synthesis rate. Neuroendocrinology 53:416-422.

Roland BL, Sawchenko PE (1993) Local origins of some GABAergic projections to the paraventricular and supraoptic nuclei of the hypothalamus in the rat. J Comp Neurol 332:123-143.

Roper P, Callaway J, Armstrong W (2004) Burst initiation and termination in phasic vasopressin cells of the rat supraoptic nucleus: a combined mathematical, electrical, and calcium fluorescence study. J Neurosci 24:4818-4831.

Sabatier N, Brown CH, Ludwig M, Leng G (2004) Phasic spike patterning in rat supraoptic neurones in vivo and in vitro. J Physiol 558:161-180.

Shuster SJ, Riedl M, Li X, Vulchanova L, Elde R (2000) The kappa opioid 
receptor and dynorphin co-localize in vasopressin magnocellular neurosecretory neurons in guinea-pig hypothalamus. Neuroscience 96:373-383.

Tribollet E, Dreifuss JJ (1981) Localization of neurones projecting to the hypothalamic paraventricular nucleus area of the rat: a horseradish peroxidase study. Neuroscience 6:1315-1328.

van den Pol AN, Wuarin JP, Dudek FE (1990) Glutamate, the dominant excitatory transmitter in neuroendocrine regulation. Science 250:1276-1278.

Wagner JJ, Terman GW, Chavkin C (1993) Endogenous dynorphins inhibit excitatory neurotransmission and block LTP induction in the hippocampus. Nature 363:451-454.

Wakerley JB, Poulain DA, Dyball RE, Cross BA (1975) Activity of phasic neurosecretory cells during haemorrhage. Nature 258:82-84.

Wakerley JB, Poulain DA, Brown D (1978) Comparison of firing patterns in oxytocin- and vasopressin-releasing neurones during progressive dehydration. Brain Res 16:425-440.

Watson SJ, Akil H, Fischli W, Goldstein A, Zimmerman E, Nilaver G, van wimersma Griedanus TB (1982) Dynorphin and vasopressin: common localization in magnocellular neurones. Science 216:85-87.

Wray S, Kusano K, Gainer H (1991) Maintenance of LHRH and oxytocin neurons in slice explants cultured in serum-free media: effects of tetrodotoxin on gene expression. Neuroendocrinology 54:327-339.

Wuarin JP, Dudek FE (1993) Patch-clamp analysis of spontaneous synaptic currents in supraoptic neuroendocrine cells of the rat hypothalamus. J Neurosci 13:2323-2331.

Yang CR, Senatorov VV, Renaud LP (1994) Organum vascularosum lamina terminalis-evoked postsynaptic responses in rat supraoptic neurones in vitro. J Physiol 477:59-74. 